\title{
Erratum to: Research progress in Asia on methods of processing laser-induced breakdown spectroscopy data
}

\author{
Yang-Min Guo ${ }^{1}$, Lian-Bo Guo ${ }^{1, \dagger}$, Jia-Ming Li $^{1}$, Hong-Di Liu ${ }^{1}$, Zhi-Hao Zhu ${ }^{1}$, \\ Xiang-You $\mathrm{Li}^{1}$, Yong-Feng $\mathrm{Lu}^{2}$, Xiao-Yan Zeng ${ }^{1}$ \\ ${ }^{1}$ Wuhan National Laboratory for Optoelectronics, Huazhong University of Science and Technology (HUST), \\ Wuhan 430074, China \\ ${ }^{2}$ Department of Electrical and Computer Engineering, University of Nebraska-Lincoln, Lincoln, NE 68588-0511, USA \\ Corresponding author. E-mail: †lbguo@mail.hust.edu.cn \\ Received March 23, 2020; accepted March 26, 2020
}

In the original publication of the article, there is one mistake in the authors' affiliations. Prof. Yong-Feng Lu's affiliation should be: Department of Electrical and Computer Engineering, University of Nebraska-Lincoln, Lincoln, NE 68588-0511, USA.

In another paper entitled "Recent technological progress in Asia from the First Asian Symposium on Laser-induced Breakdown Spectroscopy" by Lian-Bo Guo et al., one of the same mistakes also needs to be corrected. Prof. YongFeng Lu's affiliation should be: Department of Electrical and Computer Engineering, University of Nebraska-Lincoln, Lincoln, NE 68588-0511, USA.

\footnotetext{
*The online versions of the original articles can be found at: https://doi.org/10.1007/s11467-016-0604-3 (and http://journal.hep.com.cn/fop/ EN/10.1007/s11467-016-0604-3) and https://doi.org/10.1007/s11467-016-0635-9 (and http://journal.hep.com.cn/fop/EN/10.1007/s11467016-0635-9).
} 